\title{
Motivos de consulta psicológica en grupos etarios
}

Grecia Eugenia Rodríguez Navarro, ${ }^{1}$ Enrique Rosales Acevedo, ${ }^{1}$ María Dolores García Sánchez ${ }^{1}$ y Oliva Eréndira Luis Delgado ${ }^{1}$

\section{Introducción}

Por salud mental se entiende la capacidad del individuo y del grupo para interactuar con el ambiente de forma que se promueva el bienestar y las habilidades cognitivas, emocionales y relacionales (Montero, s.f.). Cuando surge un desequilibrio en la interacción de factores individuales, grupales y ambientales, hablamos de un problema de salud pública que afecta a millones de personas en el mundo (oms, 2004).

La Comisión de Comunidades Europeas (2005) informó que para el año 2020 la depresión será una de las principales causas de discapacidad laboral. Un problema de orden psicológico impacta a quien lo/a padece en distintos ámbitos y lo/a limita para gozar de bienestar; entendiendo el bienestar como la integración de factores biopsicosociales, que incorpora "sentirse bien" (bienestar subjetivo, hedonista y evaluativo) y "encontrarse bien" en términos de funcionar adecuadamente en su vida

\footnotetext{
1 Universidad Autónoma de Zacatecas.
} 
cotidiana y poder satisfacer sus necesidades (Michaelson, Abdallah, Steuer, Thompson y Marks, 2009).

\section{Planteamiento del problema}

Un motivo de consulta es la base para que el terapeuta pueda comenzar su indagación (Rodríguez, 2007). Este motivo hace referencia a las inquietudes del consultante que ocasionan que la persona acuda a terapia psicológica ya que desequilibran su vida cotidiana. Con base en los planteamientos hechos, surgen las siguientes preguntas de investigación: ¿Cuáles son los principales motivos de consulta psicológica por los que acudió la población de estudio a la UNEME-CISAME Zacatecas en el periodo 2008-2011, respecto al grupo etario?, ¿cuáles son las diferencias respecto al género en los motivos de consulta entre la población que acudió a la UNEME-CISAME Zacatecas en el periodo 2008-2011?, ¿cuál es el grupo etario predominante entre los consultantes de la UNEME-CISAME Zacatecas en el periodo 20082011? Los objetivos de este trabajo de investigación partieron de: 1) identificar los motivos de consulta psicológica más frecuentes por grupo etario, 2) identificar las diferencias genéricas en cuanto a los motivos de consulta y 3 ) hacer un perfil sociodemográfico de la población.

\section{Método}

Se llevó a cabo una investigación cuantitativa de tipo descriptiva y no experimental. La investigación realizada fue llevada a cabo en la Unidad de Especialidades Médicas Psiquiátricas-Centro Integral de Salud Mental (UNEME-CISAME), ubicada en el fraccionamiento "La Isabelica," Rinconada de la Isabelica s/n, Zacatecas, Zacatecas. Los expedientes fueron proporcionados por uno de los tres terapeutas de la unidad. 


\section{Participantes}

La población estuvo conformada por 885 participantes de entre 3 y 91 años de edad. 512 mujeres (57.85\%) y 372 hombres (42.03\%). La distribución anual fue la siguiente: en 2008: 146 consultantes; 73 mujeres y 73 hombres. En el año 2009: 225 consultantes; 125 mujeres y 100 hombres. En el año 2010: 263 consultantes; 161 mujeres y 102 hombres, y en el año 2011: 251 consultantes; 152 mujeres y 98 hombres.

\section{Instrumento}

Se utilizó la cédula de registro de la UNEME-CISAME, se extrajeron las variables sociodemográficas (edad, género, estado civil, ocupación, escolaridad y referido por). También se obtuvo la variable dependiente (motivo de consulta psicológica).

\section{Procedimiento}

El procedimiento consistió en la revisión de expedientes, la eliminación de expedientes incompletos, la clasificación anual y la sistematización en base de datos. Consecutivamente, el análisis de datos se realizó con el programa estadístico-informático Statistical Package for the Social Sciences y se llevó a cabo un análisis estadístico-descriptivo.

Partiendo de la Psicología del Desarrollo, se tomó como referentes a Papalia, Wandkos y Dustin (2005). Sobre la división etaria se agrupó la infancia, la niñez temprana e intermedia en el grupo de los 1-10 años, la adolescencia de los 11-20, se dividió la edad adulta temprana en dos grupos; de los 21-30 y de los 31-40. La edad adulta intermedia se dividió de los 41-50 y de los 51-60 años. Por último, la edad adulta tardía se concentró en el grupo de los 60 y más. Al desglosar las etapas propuestas por los autores se buscó brindar resultados por grupos de 10 años para dar a conocer los principales motivos de consulta en cada uno. 


\section{Resultados}

A continuación, se describen los principales resultados concernientes al grupo etario predominante y el principal motivo de consulta de este grupo con base en la población anual. En el año 2008 el grupo etario de los 21-30 años obtuvo mayor asistencia con un porcentaje de $27.40 \%$, los principales motivos de consulta fueron agresividad (17.05\%) y depresión (17.05\%). Para el año 2009, dos grupos etarios predominaron; el grupo de los 11-20 años, con un porcentaje de $20 \%$, el principal motivo de consulta fue molestias físicas (17.78\%). El grupo etario de los 21-30 también obtuvo una asistencia de $20 \%$ y el principal motivo de consulta fue la ansiedad, con un porcentaje de $28.88 \%$. En cuanto al año 2010, el grupo etario predominante fue el de los 31-40 años, con 19.39\%; el principal motivo de consulta fue la depresión (25.49\%). Por último, se analizó el año 2011, en el que el grupo de los $11-20$ años tuvo un porcentaje de $24.30 \%$, y el principal motivo de consulta fue problemas de conducta (22.95\%).

En relación con el género y los motivos de consulta principales, cabe mencionar que los porcentajes se obtuvieron con base en las frecuencias absolutas (f) de cada motivo de consulta resultante en cada año; en el año 2008, el género femenino acudió principalmente por depresión (80\%) y el masculino por agresividad (70.60\%). En 2009, tanto mujeres (68.18\%) como hombres (31.80\%) acudieron primariamente por ansiedad. Para el año 2010, el género femenino asistió por depresión (87.27\%) y el masculino por ansiedad (41.67\%). En 2011, las mujeres reportaron principalmente depresión (80\%) y los hombres asistieron por problemas de conducta (69.57\%)

En cuanto al perfil sociodemográfico, en 2008 la población se constituyó del mismo número de mujeres y de hombres (50\%), casados/as (54.11\%), residentes en la capital (Zacatecas) (30.14\%), con secundaria interrumpida (16.49\%), de ocupación al hogar (27.40\%) y referidos por otro paciente. Para el año 2009 asistió mayormente el género femenino (55.56\%), casadas (44.44\%), residentes en Zacatecas (37.78\%), con primaria inte- 
rrumpida (18.67\%), de ocupación al hogar (32\%) y referidas por otro paciente (34.22\%).

En el año 2010 acudió un mayor número de mujeres (61.21\%), casadas (41.445), residentes en Zacatecas (34.6\%), con primaria interrumpida (17.86\%), de ocupación al hogar (31.18\%) y referidas por otro paciente (37.26\%). Por último, en el año 2011, la población se conformó principalmente por mujeres (60.59\%), solteras/os (44.62\%), residentes en Zacatecas (42.63\%), con primaria interrumpida (15.94\%), dedicadas al hogar $(29.48 \%)$ y referidas por otro paciente (28.29\%).

\section{Discusión}

A partir de los resultados obtenidos, inferimos la prevalencia de dos grupos etarios en la comparación anual del periodo 20082011; el grupo de los 11-20 y de los 21-30. El grupo de los 1120 años acudió principalmente por molestias físicas y problemas de conducta, resultados acordes a los encontrados por Navarro, Meléndez, Sales y Sancerni (2012), ya que los trastornos psicológicos en la población del mismo rango etario, en su estudio, fueron los problemas de conducta. Tomemos en cuenta que son los padres o tutores quienes llevan a terapia psicológica a los hijos.

El grupo etario de los 21-30 años obtuvo como principales motivos de consulta agresividad, ansiedad y depresión, este grupo corresponde a la edad adulta temprana (Papalia, Wandkos y Dustin, 2005). En esta etapa los jóvenes atraviesan por diversas situaciones como estar casado, estar divorciándose, ser estudiante, ser egresado y desempleado; asimismo, se enfrentan a diferentes condiciones socioeconómicas, carecen de redes sociales, padecen de alcoholismo, tienen inseguridad, entre otras. Estas variables forman parte de los padecimientos psicológicos de los jóvenes. En este sentido, la Secretaría de Salud de Medellín (2009), en su encuesta nacional, encontró entre jóvenes de 12-19 años una tendencia elevada a la ansiedad, ya que sólo $6.9 \%$ de los entrevistados no la reportaron. Por su parte, Vicente, Rioseco, Saldivia, Kohn y Torres (2005) llevaron 
a cabo el Estudio Chileno de Prevalencia de Patología Psiquiátrica, encontrando que 1 de cada 3 encuestados, es decir, $36 \%$, presentó un trastorno psiquiátrico en el último año.

En cuanto a las diferencias de género para este mismo periodo (2008-2011), prevaleció como principal motivo de consulta la depresión en el género femenino, mientras que en el género masculino los motivos variaron: agresividad, ansiedad y problemas de conducta, esto acorde a datos encontrados por Muñoz y Novoa (2011) en su investigación. Las mujeres acudieron a terapia por respuestas emocionales intensas y los hombres por comportamientos agresivos. Kendler, Thorton y Prescott (2001) ya expresaban estas diferencias, su estudio reveló que los estresores en la mujer se relacionan con pérdida de relaciones y en hombres con pérdida de objetos y metas.

Estudios previos (García, Lozano, Luis-Delgado y Zavala, 2010; Secretaría de Salud de Zacatecas, 2013) aluden a las diferencias genéricas, reportando mayor asistencia del género femenino y la tendencia a la depresión. Las diferencias genéricas deben ser incluidas como categorías que permitan el entendimiento del contexto para posteriormente implementar estrategias y planes a nivel preventivo en comunidades determinadas para partir de las necesidades de esta misma.

\section{Conclusiones}

- En el año 2008, el grupo etario de mayor asistencia fue el de los 21-30 años de edad, la agresividad y la depresión fueron los motivos principales.

- En el año 2009, el grupo de los 11-20 años reportó como principal motivo las molestias físicas y el grupo de los 2130 años la ansiedad.

- Para el año 2010, el grupo etario de mayor asistencia fue el de los 31-40 años, reportando la ansiedad como principal motivo.

- Para el año 2011, el grupo etario de los 11-20 años fue el de mayor asistencia y acudió por problemas de conducta. 
- En el año 2008, el género femenino acudió principalmente por depresión y el masculino por agresividad.

- En el año 2009, tanto mujeres como hombres asistieron primariamente por ansiedad.

- Para el año 2010, el género femenino acudió por depresión y el masculino por ansiedad.

- En el año 2011, las mujeres acudieron por depresión y los hombres por problemas de conducta.

- El perfil sociodemográfico se constituyó por mujeres de entre los 11-40 años, casadas, dedicadas al hogar, residentes en la capital, Zacatecas, con primaria interrumpida y referidas por otro paciente.

\section{Referencias}

Comisión de Comunidades Europeas. (2005). Libro verde: mejorar la salud mental de la población. Hacia una estrategia de la unión europea en materia de salud mental. Bruselas: сом.

García, S.M.D., Lozano, R.G., Luis-Delgado, O.E. y Zavala, R.J. (2010). Consulta psicológica en Zacatecas: principales motivos. En R.I. Reyes (Ed.), Zacatecas y su contexto actual: Reflexiones desde las ciencias sociales sobre el desarrollo social (75-94). México: Taberna Librería Editores.

Kendler, E.R., Thorton, L.M. y Prescott, C.A. (2001). Gender Differences in the Rates of Exposure to Stress Full Life Events: Sensivity to Their Depressogenic Effects. American Journal of Psychiatric, 154(4), 58-93. Recuperado de http://www.ncbi.nlm.nih.gov/pudmed/11282693.

Michelson, J., Abdallah, S., Steuer, N., Thompson, S. y Marks, N. (2009). National Accounts of Well-being. Londres: Nef.

Montero, P.I. (s.f.). Salud mental. Programa de formadores/as en perspectiva de género en salud. Universidad de Valencia. Recuperado de http://www.msssi.gob.es/organizacion/ sns/planCalidadSNS/pdf/equidad/06modulo05.pdf.

Muñoz, M.A.M. y Novoa, G.M.M. (2011). Motivos de consulta e hipótesis clínicas explicativas. Terapia Psicológica, 30(1), 
25-36. Recuperado de http://www.redalyc.org/articulo. ao?id=78523000003.

Navarro-Prado, E., Meléndez, M.J.C., Sales, G.A. y Sancerni, B.M.D. (2012). Desarrollo infantil y adolescente: trastornos mentales. Psicothema, 24(3), 377-383. Recuperado de http://www.psicothema.com/pdf/4026.pdf.

Organización Mundial de la Salud. (2004). Intervenir en Salud Mental. World Health Organization. Ginebra: oms.

Papalia, D.E., Wandkos, O.S. y Duskin, F.R. (2005). Psicología del Desarrollo: De la infancia a la adolescencia. México: Mc Graw Hill.

Rodríguez, D. (2007). Motivos de consulta y perspectiva clínica constructivista. Diversitas, 3(2), 239-247. Recuperado de www.redalyc.org/pdf/679/67930205.pdf.

Secretaría de Salud de Medellín. (2009). Segundo Estudio de Salud Mental del adolescente. Colombia: SSM.

Secretaría de Salud de Zacatecas. (2013). Diagnóstico del Centro Integral de Salud Mental. UNEME-CISAME Zacatecas (Informe No. 4). Zacatecas, México: UNEME-CISAME.

Vicente, B., Rioseco, P., Saldivia, S., Khon, R. y Torres, S. (2005). Estudio Chileno de Prevalencia de Patología Psiquiátrica. Revista Médica de Chile, 34(4). Recuperado de http://www. scielo.org.com/acielo.php?script=sci_arttwX\&pid=S0034. 